\title{
Extreme tadpoles: The morphology of the fossorial megophryid larva, Leptobrachella mjobergi
}

\author{
Alexander Haas ${ }^{\mathrm{a}, *}$, Stefan Hertwig ${ }^{\mathrm{b}}$, Indraneil Das ${ }^{\mathrm{c}}$ \\ ${ }^{a}$ Biozentrum Grindel und Zoologisches Museum, Martin-Luther-King-Platz 3, D-20146 Hamburg, Germany \\ ${ }^{\mathrm{b}}$ Institut für Spezielle Zoologie und Evolutionsbiologie, Erbertstr. 1, D-07743 Jena, Germany \\ ${ }^{\mathrm{c}}$ Institute of Biodiversity and Environmental Conservation, Universiti Malaysia Sarawak, 94300 Kota Samarahan, Sarawak, \\ Malaysia
}

Received 15 June 2005; received in revised form 9 September 2005; accepted 30 September 2005

\begin{abstract}
The bizarre larvae of Leptobrachella mjobergi are fossorial and live in the gravel beds of small streams. These tadpoles are vermiform in body shape. Here we present details on their skeleton and musculature, particularly of the head. The entire cranium and its associated musculature are reconstructed in three dimensions from serial histological sections. The hyobranchial apparatus is highly reduced. The head of the L. mjobergi larva is more mobile than in other anuran species. This mobility can largely be ascribed to the exclusion of the notochord from the cranial base and an articulation of the foramen magnum floor with the atlas of the tadpole. The articulation is unique among anuran species, but design parallels can be drawn to salamanders and the articulation between atlas and axis in mammals. In L. mjobergi, the atlas forms an anterior dens that articulates with the basal plate in an accessory, third occipital articular face. The muscle arrangements deviate from the patterns found in other tadpoles: For instance, epaxial and ventral trunk muscles reach far forward onto the skull. The post-cranial skeleton of L. mjobergi is considerably longer than that of other anurans: it comprises a total of 35 vertebrae, including more than 20 post-sacral perichordal centra. Despite a number of features in cranial and axial morphology of L. mjobergi, which appear to be adaptations to its fossorial mode of life, the species clearly shares other features with its megophryid and pelobatid relatives.
\end{abstract}

(C) 2005 Elsevier GmbH. All rights reserved.

Keywords: Pelobatidae; Megophryidae; Anuran larvae; Cranial morphology; Cranial musculature; Fossorial tadpole

\section{Introduction}

With over 5000 extant species, anurans are the most successful group of lissamphibians (Frost, 2004). The remarkable diversity of reproductive modes, larval forms and adaptive strategies exhibited by anurans are unquestionably major determinants of their evolution-

\footnotetext{
${ }^{*}$ Corresponding author.

E-mail address: alexander.haas@uni-hamburg.de (A. Haas).
}

ary success (McDiarmid and Altig, 1999). Ecomorphological guilds have been described containing distantly related species indicating much of convergence in the evolution of adaptive types and resource use. In different regions of the world equivalent microhabitats are used by similar larval ecomorphs belonging to different frog taxa (Orton, 1953; Altig and Johnston, 1989; Altig and McDiarmid, 1999a).

An elongate and slender larval ecomorph has evolved in the megophryid genus Leptobrachella (Inger, 1983, 